\title{
Reproductive Health Services for Women During COVID-19 Pandemic
}

\author{
Farwa Rizvi ${ }^{1 *}$ and Rabab Rizvi ${ }^{2}$ \\ Faculty of Health, School of Health and Social Development, Deakin University, Australia \\ Consultant Psychiatrist Health Link Now, and Insight Tele-psychiatry, Texas, USA
}

*Corresponding author: Farwa Rizvi, PhD candidate, Faculty of Health, School of Health and Social Development, Deakin University, 221 Burwood Highway, Burwood 3125, VIC Australia.

To Cite This Article: Farwa Rizvi, Rabab Rizvi, Reproductive Health Services for Women During COVID-19 Pandemic. 2020 - 10(1). AJBSR. MS.ID.001469. DOI: 10.34297/AJBSR.2020.10.001469.

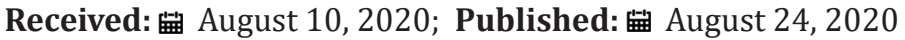

\section{Opinion}

Millions of women across the world risk losing access to essential reproductive health services during the prevalent COVID-19 pandemic. The global and national level healthcare contexts are rapidly changing, and strongly influencing the sexual and reproductive health (SRH) and rights of adolescent girls and women [1]. Sustainable SRH services are of paramount importance in this current pandemic scenario. The SRH rights include an individual's overall well-being for the SRH and reproductive body and considered imperative for the personal, social and economic growth [2]. The Sustainable Development Goals (SDGs) 3 and 5 are concerned with SRH rights pertaining to gender equality, women empowerment, accessible family planning services and contraception use [3]. The deep-rooted, gender-based inequalities for females in accessing SRH care have come to the forefront during the COVID-19 pandemic. Disrupted SRH services, gender inequality, and gender-based violence threatens to become a pandemic within the existing pandemic according to the United Nations Population Fund (UNFPA) [1,4].

The World Health Organization [5] in March 2020 issued guidelines to make SRH services as priority services during the current pandemic to reduce the burden of maternal morbidity and mortality. Many countries have implemented tough measures for lockdown and travel restrictions in a bid to control the spread of infection [6]. However, during these restrictions, the SRH service providers were also closed, as these were not classified as essential health services. These critical SRH services including abortion, and emergency contraception or regular contraception have become difficult to obtain due to travel restrictions, interrupted supply chains, and excessive load on the healthcare infrastructure. Some of the countries where contraception services have been negatively affected include Zambia, Sudan, Uganda, Ghana, Zimbabwe, Pakistan, Sri-Lanka, Malaysia, El Salvador, Colombia, and Germany $[7,8,9]$.

There is an estimated $10 \%$ decrease in the use of short-acting contraceptives, and long-acting reversible contraceptives (LARCs) including subdermal implants and intra-uterine contraceptive devices (IUCDs) in the low-to-middle-income countries (LMICs) [9]. This decrease in use of modern contraceptives could potentially result in 15 million unintended pregnancies in one year [9]. The revised global recommendations include an extended use of LARCs, and additional 12 months' supply of combined oral contraception pills (COCP) for women who face barriers to attend the clinic during the COVID-19 pandemic [10]. More women in the United States have reported a change in their fertility preferences, wanting to delay pregnancy (birth spacing) or wanting fewer children (limiting pregnancies) during the current pandemic. Women with lower income are more likely to report this change in their fertility preferences, yet many women are having difficulty getting their birth control contraception [11].

In many countries, the access to contraception especially LARCs was initially affected during the pandemic due to lockdown, movement restrictions, an initial lack of personal protective equipment (PPE) and a shift from in-person to telehealth consultations. In Australia, the government quickly responded by expanding the telehealth services for access to contraception which were bulk billed to Medicare [4]. Utilization 
of telehealth consultation in Australia has helped maintain access to contraception during this crisis $[6,12]$. The use of telehealth contraception consultations has paved the way for provision of services to the hard-to-reach groups including ethnic minorities and those in rural areas. The equitable services for abortion have been negatively affected worldwide. It is of paramount importance to consider abortion as an essential service during the pandemic [12]. The WHO classifies abortion as essential to women's rights and health, but anti-choice organizations are using this pandemic as a guise and urging governments to prohibit reproductive health services.

During this current pandemic, many states in US have declared time-sensitive abortions as non-essential, or as an elective health procedure. By May 2020, at least 11 states have tried to restrict access to abortion [13]. The global telehealth consultations for early and medical abortions have increased by $25 \%$ since the beginning of COVID-19 pandemic [4]. This indicates an effective way of dealing with the physical and financial barriers to obtaining safe abortion services. suggests that pre-abortion check-up can be done easily through Skype as a telehealth consultation [14]. Whereas UK has rapidly adopted telehealth-assisted abortion at home, many LMICs could not make the telehealth consultation work effectively due to lack of robust healthcare infrastructure. The exceptions include countries such as South-Africa, Ethiopia and Nepal, where telehealth consultations have been already in place for dispensing of medical bills and nurses providing medical abortion at home [4].

\section{Recommendations}

Some recommendations include; Ensuring robust telehealth services for contraception and early abortion, removing physical, financial and policy barriers to access contraception methods including multiple prescriptions for supply. There is a need to provide SRH services and sustainable supply of contraception methods including LARCs (subdermal implants and IUCDs) and short-acting contraception methods during the current pandemic. These SRH services also include training women to utilize injectable contraceptives at home and utilizing the post-partum period to offer permanent contraception procedures [15]. In addition, pharmacists and nurses could be trained to perform LARC insertion, either subdermal implant in the underarm or the IUCD insertion. Abortions need to be prioritized as essential services, as these are time-sensitive, and women could be assisted for medical abortions at home in the early stages via telehealth as priority consultations. We recommend using telehealth services to provide sustainable SRH and family planning services to adolescent girls and women for effectively preventing unintended pregnancies to reduce the maternal morbidity and mortality. This is in lieu of the global commitment to adhere to Sustainable Development Goals for SRH rights, gender equality and women empowerment.

\section{References}

1. SchaafM, BoydellV, Van BelleS, Brinkerhoff DW, GeorgeA, et al. (2020) 'Accountability for SRHR in the context of the COVID-19 pandemic'. Sexual and Reproductive Health Matters 28(1): 1779634.

2. StarrsAM, EzehAC, BarkerG, BasuA, BertrandJT, et al. (2018) 'Accelerate progress - sexual and reproductive health and rights for all: report of the Guttmacher-Lancet Commission'.The Lancet 391(10140): 26422692.

3. UNFPA (2019) Sexual and Reproductive Health and Rights: An Essential Element of Universal Health Coverage. Background document for the Nairobi Summit on ICPD 25 - Accelerating the promise 44.

4. Cousins S (2020) 'COVID-19 has "devastating" effect on women and girls'. The Lancet 396(10247): 301-302.

5. WHO (2020) Coronavirus disease (COVID-19) and Sexual and Reproductive Health?

6. WHO (2020) COVID-19: operational guidance for maintaining essential health services during an outbreak: interim guidance World Health Organization?

7. Church K, Gassner J, Elliott M (2020)'Reproductive health under Covid-19-challenges of responding in a global crisis'. Sexual and Reproductive Health Matters p. 1-3.

8. Kumar N (2020) 'COVID 19 era: a beginning of upsurge in unwanted pregnancies, unmet need for contraception and other women related issues'. The European Journal of Contraception \& Reproductive Health Care 25(4): 323-325.

9. Riley T, Sully E, Ahmed Z, Biddlecom A (2020) 'Estimates of the potential impact of the COVID-19 pandemic on sexual and reproductive health in low-and middle-income countries'. Int Perspect Sex Reprod Health 46: 73-76.

10. FSRH (2020) FSRH CEU recommendation on extended use of the etonogestrel implant and 52mg levonorgestrel-releasing intrauterine system during COVID restrictions. Faculty of Sexual and Reproductive Healthcare of the Royal College odf Obstetricians and Gynaecologists London.

11. Lindberg L, VandeVusse A, Muelle J, Kirstein M (2020) 'Early Impacts of the COVID-19 Pandemic: Findings from the 2020 Guttmacher Survey of Reproductive Health Experiences'. Guttmacher Institute 24.

12. RANZCOG (2020) Advice to Obstetricians and Gynecologists Melbourne Australia.

13. Jones RK, Lindberg L, Witwer E (2020) 'COVID-19 Abortion Bans and Their Implications for Public Health'. Perspectives on Sexual and Reproductive Health 52(2): 65-68.

14. Berer M (2020) 'Reconceptualizing safe abortion and abortion services in the age of abortion pills: A discussion paper'. Best Practice \& Research Clinical Obstetrics \& Gynaecology 63: 45-55.

15. NandaK, LebetkinE, SteinerMJ, YacobsonI, Dorflinger LJ, et al. (2020) Contraception in the Era of COVID-19. Global Health: Science and Practice 8(2). 